\title{
On Grids in Topological Graphs
}

\author{
Eyal Ackerman* Jacob Fox ${ }^{\dagger} \quad$ János Pach$\quad$ Andrew Suk ${ }^{\S}$
}

\begin{abstract}
A topological graph is a graph drawn in the plane with vertices represented by points and edges as arcs connecting its vertices. A $k$-grid in a topological graph is a pair of edge subsets, each of size $k$, such that every edge in one subset crosses every edge in the other subset. It is known that for a fixed constant $k$, every $n$-vertex topological graph with no $k$-grid has $O(n)$ edges. We conjecture that this remains true even when: (1) considering grids with distinct vertices; or (2) all edges are straight-line segments and the edges within each subset of the grid are required to be pairwise disjoint. These conjectures are shown to be true apart from $\log ^{*} n$ and $\log ^{2} n$ factors, respectively. We also settle the conjectures for some special cases, including the second conjecture for convex geometric graphs. This result follows from a stronger statement that generalizes the celebrated Marcus-Tardos Theorem on excluded patterns in 0-1 matrices.
\end{abstract}

\section{Introduction}

The intersection graph of a set $\mathcal{C}$ of geometric objects has the members of $\mathcal{C}$ as its vertex set and an edge between every pair of objects with a nonempty intersection. The problems of finding maximum independent set and maximum clique in the intersection graph of geometric objects have received a considerable amount of attention in the literature due to their applications in VLSI design [HM85], map labeling [AKS98], frequency assignment in cellular networks [M97], and elsewhere. Here we study the intersection graph of the edge set of graphs that are drawn in the plane. It is known that if this intersection graph does not contain a large complete bipartite subgraph, then the number of edges in the original graph is small. We show that this remains true even under some very restrictive conditions.

A topological graph is a graph drawn in the plane with points as vertices and edges as arcs connecting its vertices. The arcs are allowed to cross, but they may not pass through vertices except for their endpoints. We only consider graphs without parallel edges or self-loops. A topological graph is simple if every pair of its edges intersect at most once. If the edges are drawn as straight-line segments, then the graph is geometric. A geometric graph is convex if its vertices are in convex position, that is, they are the vertices of a convex polygon.

Given a topological graph $G$, the intersection graph of $E(G)$ has the edge set of $G$ as its vertex set, and an edge between every pair of crossing edges. Note that we consider the edges of $G$ as open curves, therefore, edges that intersect only at a common vertex are not adjacent in the intersection graph. A complete bipartite subgraph in the intersection graph of $E(G)$ corresponds to a grid structure in $G$.

\footnotetext{
${ }^{*}$ Department of Computer Science, Freie Universität Berlin, Takustraße 9, 14195 Berlin, Germany. Email: eyal@inf.fu-berlin.de. Supported by a fellowship from the Alexander von Humboldt Foundation.

${ }^{\dagger}$ Department of Mathematics, Princeton, Princeton, NJ 08544. Email: jacobfox@math.princeton.edu. Research supported by an NSF Graduate Research Fellowship and a Princeton Centennial Fellowship.

${ }^{\ddagger}$ City College, CUNY and Courant Institute, NYU, New York, NY. Email: pach@cims.nyu.edu. Supported by NSF Grant CCF-05-14079, and by grants from NSA, PSC-CUNY, the Hungarian Research Foundation OTKA, and BSF.

${ }^{\S}$ Department of Mathematics, Courant Institute of Mathematical Sciences, New York University, 251 Mercer St., New York, NY 10012. Email: suk@cims.nyu.edu
} 


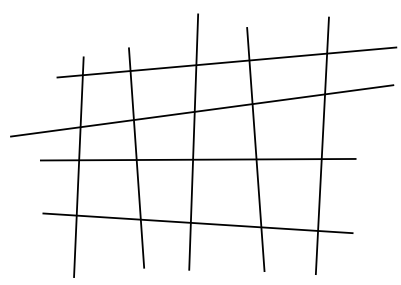

Figure 1: a "natural" grid

Definition $1.1 A(k, l)$-grid in a topological graph is a pair of edge subsets $E_{1}, E_{2}$ such that $\left|E_{1}\right|=k$, $\left|E_{2}\right|=l$, and every edge in $E_{1}$ crosses every edge in $E_{2} . A k$-grid is an abbreviation for a $(k, k)$-grid.

Theorem 1.2 ([PPST05]) Given fixed constants $k, l \geq 1$ there exists another constant $c_{k, l}$, such that any topological graph on $n$ vertices with no $(k, l)$-grid has at most $c_{k, l}$ n edges.

The proof of Theorem 1.2 in [PPST05] actually guarantees a grid in which all the edges of one of the subsets are adjacent to a common vertex. For two recent and different proofs of Theorem 1.2 see [FPT08] and [FP08b]. Tardos and Tóth [TT07] extended the result in [PPST05] by showing that there is a constant $c_{k}$ such that a topological graph on $n$ vertices and at least $c_{k} n$ edges must contain three subsets of $k$ edges each, such that every pair of edges from different subsets cross, and for two of the subsets all the edges within the subset are adjacent to a common vertex.

Note that according to Definition 1.1 the edges within each subset of the grid are allowed to cross or share a common vertex, as is indeed required in the proofs of [PPST05] and [TT07]. However, a drawing similar to Figure 1 usually comes to mind when one thinks of a "grid". That is, we would like every pair of edges within each subset of the grid to be disjoint, i.e., neither to share a common vertex nor to cross. We say that a $(k, l)$-grid formed by edge subsets $E_{1}$ and $E_{2}$ is natural if the edges within $E_{1}$ are pairwise disjoint, and the edges within $E_{2}$ are pairwise disjoint.

Conjecture 1.3 Given fixed constants $k, l \geq 1$ there exists another constant $c_{k, l}$, such that any simple topological graph $G$ on $n$ vertices with no natural $(k, l)$-grid has at most $c_{k, l}$ n edges.

Note that it is already not trivial to show that an $n$-vertex geometric graph with no $k$ pairwise disjoint edges has $O(n)$ edges (see [PT94] and [T00]). Moreover, it is an open question whether a simple topological graph on $n$ vertices and no $k$ disjoint edges has $O(n)$ edges (the best upper bound, due to Pach and Tóth [PT05], is $\left.O\left(n \log ^{4 k-8} n\right)\right)$. Therefore, a proof of Conjecture 1.3 is probably hard to obtain. Here we prove the following bounds for geometric and simple topological graphs with no natural $k$-grids.

\section{Theorem 1.4}

(i) An n-vertex geometric graph with no natural $k$-grid has $O\left(k^{2} n \log ^{2} n\right)$ edges.

(ii) An n-vertex simple topological graph with no natural $k$-grid has $O\left(n \log ^{4 k-6} n\right)$ edges.

We also settle Conjecture 1.3 for two special cases.

Theorem 1.5 An n-vertex simple topological graph with no natural $(2,1)$-grid has $O(n)$ edges.

Theorem 1.6 Given a fixed constant $k \geq 1$ there exists another constant $c_{k}$, such that any convex geometric graph on $n$ vertices with no natural $k$-grid has at most $c_{k} n$ edges. 
Note that Theorem 1.5 is the first nontrivial case since an $n$-vertex topological graph with no $(1,1)$-grid is planar and hence has at most $3 n-6$ edges, for $n>2$. Instead of Theorem 1.6 we actually prove a stronger statement that generalizes the Marcus-Tardos Theorem. This theorem, conjectured by Füredi and Hajnal [FH92] and later proved by Marcus and Trados [MT04], settled the famous Stanley-Wilf Conjecture. See Section 4 for more details on the Marcus-Tardos Theorem and its connection to convex geometric graphs.

Conjecture 1.3 is clearly false for (not necessarily simple) topological graphs: the complete graph can be drawn as a topological graph in which every pair of edges intersect (at most twice [PT05]). Therefore, for topological graphs we have to settle for only one of the components of "disjointness".

Conjecture 1.7 Given fixed constants $k, l \geq 1$ there exists another constant $c_{k, l}$, such that any topological graph on $n$ vertices with no $(k, l)$-grid with distinct vertices has at most $c_{k, l}$ n edges.

This conjecture is shown to be true for $l=1$.

Theorem 1.8 An n-vertex topological graph with no $(k, 1)$-grid with distinct vertices has $O(n)$ edges.

For the general case we provide a slightly superlinear upper bound.

Theorem 1.9 Every $n$-vertex topological graph with no $k$-grid with distinct vertices has at most $c_{k} n \log ^{*} n$ vertices, where $c_{k}=k^{O(\log \log k)}$ and $\log ^{*}$ is the iterated logarithm function.

Note that $c_{k}$ is just barely superpolynomial in $k$.

Organization. The rest of this paper is organized as follows. We discuss topological graphs with no grids with distinct vertices in Section 2. In Section 3 we prove the bounds for the number of edges in simple topological graphs with no natural grids. Convex geometric graphs are considered in Section 4, where we prove Theorem 1.6 as well as some tighter bounds for some special cases. We systematically omit floor and ceiling signs whenever they are not crucial for the sake of clarity of presentation. We also do not make any serious attempt to optimize absolute constants in our statements and proofs. All logarithms in this paper are base 2. Due to space limitations, some of the proofs are omitted and some appear in the appendix.

\section{Grids on distinct vertices}

In this section we prove Theorems 1.9 and 1.8 .

\subsection{Topological graphs with no $k$-grid with distinct vertices}

Here we prove Theorem 1.9. We use the following three results from three different papers.

Lemma 2.1 ([FP08]) Every string graph with $m$ vertices and $\epsilon m^{2}$ edges contains the complete bipartite graph $K_{t, t}$ as a subgraph with $t \geq \epsilon^{c_{1}} \frac{m}{\log m}$, where $c_{1}$ is an absolute constant.

The pair-crossing number pair-cr $(G)$ of a graph $G$ is the minimum possible number of unordered pairs of crossing edges in a drawing of $G$. The bisection width, denoted by $b(G)$, is defined for every simple graph $G$ with at least two vertices. It is the smallest nonnegative integer such that there is a partition of the vertex set $V=V_{1} \dot{\cup} V_{2}$ with $\frac{1}{3} \cdot|V| \leq V_{i} \leq \frac{2}{3} \cdot|V|$ for $i=1,2$, and $\left|E\left(V_{1}, V_{2}\right)\right|=b(G)$. We will use the following result of Kolman and Matoušek [KM04] which relates the pair-crossing number and the bisection width of a graph. 
Lemma 2.2 ([KM04]) There is an absolute constant $c_{2}$ such that if $G$ is a graph with $n$ vertices of degrees $d_{1}, \ldots, d_{n}$, then

$$
b(G) \leq c_{2} \log n\left(\sqrt{\text { pair-cr }(G)}+\sqrt{\sum_{i=1}^{n} d_{i}^{2}}\right) .
$$

We use the following upper bound on the number of edges of a topological graph with no $h$ pairwise crossing edges.

Lemma 2.3 ([FP08a]) If a topological graph with $n$ vertices has no $h$ pairwise crossing edges, then it has at most $n(\log n)^{c_{3} \log h}$ edges.

Let $h(k)$ be the minimum $h$ such that if a collection $C$ of $h$ pairwise intersecting curves is such that each of the curves is partitioned into one or two subcurves, then there are $k$ subcurves intersecting $k$ other subcurves, and these $2 k$ subcurves come from distinct curves in $C$. Note that $h(1)=2$.

Lemma 2.4 For $k \geq 2$, we have $h(k) \leq c_{4} k \log k$ for some absolute constant $c_{4}$.

Proof. Let $h=c_{4} k \log k$, where $c_{4}=16^{c_{1}+1}$, where $c_{1}$ is the absolute constant in Lemma 2.1. For each curve $\gamma \in C$, randomly pick one of the at most two subcurves to keep. For each pair $\gamma, \gamma^{\prime} \in C$, there is a probability at least $1 / 4$ that the subcurve of $\gamma$ we pick intersects the subcurve of $\gamma^{\prime}$ we pick. So the expected number of intersecting pairs of curves is at least $\frac{1}{4}\left(\begin{array}{l}h \\ 2\end{array}\right)$. So there is a collection $C^{\prime}$ consisting of one subcurve of the at most two subcurves for each curve such that the number of intersecting pairs of curves in $C^{\prime}$ is at least $\frac{1}{4}\left(\begin{array}{c}h \\ 2\end{array}\right)$. Since $C^{\prime}$ has cardinality $h$ and at least $\frac{1}{4}\left(\begin{array}{c}h \\ 2\end{array}\right) \geq \frac{1}{16} h^{2}$ intersecting subcurves, then by Lemma 2.1, the intersection graph of $C^{\prime}$ contains a complete bipartite graph with parts of size

$$
\left(\frac{1}{16}\right)^{c_{1}} \frac{h}{\log h} \geq k
$$

since we picked $c_{4}$ sufficiently large.

Let $f_{k}(n)$ denote the maximum number of edges of a topological graph with $n$ vertices and no $k$-grid with distinct vertices. In Theorem 1.9 , we prove the upper bound $f_{k}(n) \leq c_{k} n \log ^{*} n$. It will be helpful to consider a related function. Let $f_{k}(n, \Delta)$ denote the maximum number of edges of a topological graph with $n$ vertices, maximum degree at most $\Delta$, and no $k$-grid with distinct vertices.

We collect several useful lemmas before proving Theorem 1.9. For a graph $G$ and vertex sets $A$ and $B$, let $e_{G}(A)$ denote the number of edges with both vertices in $A$ and $e_{G}(A, B)$ denote the number of pairs $(a, b) \in A \times B$ that are edges of $G$.

Lemma 2.5 There is an absolute constant $c$ such that if $\Delta=(\log n)^{c \log k} k^{c \log \log k}$, then

$$
f_{k}(n) \leq f_{k}(n, \Delta)+k^{c \log \log k} n .
$$

Proof. See Appendix A. that

Let $d_{k}(n)=\max _{n^{\prime} \leq n} f_{k}\left(n^{\prime}\right) / n^{\prime}$ and $d_{k}(n, \Delta)=\max _{n^{\prime} \leq n} f_{k}\left(n^{\prime}, \Delta\right) / n^{\prime}$. Lemma 2.5 demonstrates

$$
d_{k}(n) \leq d_{k}(n, \Delta)+k^{c \log \log k}
$$


where $\Delta=k^{c \log \log k}(\log n)^{c \log k}$. Note that a triangulated planar graph with $n$ vertices has $3 n-6$ edges, so $d_{1}(n)=3-\frac{6}{n}$ for $n \geq 3$, so $d_{k}(n) \geq 1$ for $n \geq 3$. By Lemma 2.3, we have

$$
d_{k}(n) \leq(\log n)^{c_{3} \log 2 k}
$$

since $2 k$ pairwise crossing edges in a topological graph has a $k$-grid with distinct vertices. We will improve this bound significantly.

Lemma 2.6 There are absolute constants $c_{9}$ and $c_{10}>0$ such that for each $k, n$ and $\Delta$ with $\Delta \geq k$ and $n \geq \Delta^{c_{9}}$, there is $n_{1} \leq 2 n / 3$ such that

$$
d_{k}\left(n_{1}, \Delta\right) \geq d_{k}(n, \Delta)\left(1-n^{-c_{10}}\right) .
$$

Proof. Let $G$ be a topological graph with at most $n$ vertices, maximum degree at most $\Delta$, and no $k$-grid with distinct vertices which has maximum possible average degree among all such topological graphs. Without loss of generality, we may suppose that the number of vertices of $G$ is actually $n$, and let $m=f_{k}(n, \Delta)$. Since each vertex has degree at most $\Delta$, then $G$ does not contain a $k \Delta$-grid. Let the number of crossing pairs of edges of $G$ be $\epsilon m^{2}$, so the underlying graph of $G$ has pair-crossing number at most $\epsilon m^{2}$. By Lemma 2.1, $G$ has an $\ell$-grid with $\ell \geq \epsilon^{c_{1}} \frac{m}{\log m}$. Therefore, we have the inequality $\epsilon^{c_{1}} \frac{m}{\log m} \leq k \Delta$, and we get $\epsilon \leq m^{-\frac{2}{3 c_{1}}}$, where we use $k \Delta \leq m^{1 / 6}$ and $\log m \leq m^{1 / 6}$. By Lemma 2.2, there is an absolute constant $c_{2}$ such that if $d_{1}, \ldots, d_{n}$ is the degree sequence of $G$, then

$$
\begin{aligned}
b(G) & \leq c_{2} \log n\left(\sqrt{\text { pair-cr }(G)}+\sqrt{\sum_{i=1}^{n} d_{i}^{2}}\right) \leq c_{2} \log n\left(\epsilon^{1 / 2} m+\Delta \sqrt{n}\right) \\
& \leq c_{2} \log n\left(m^{1-\frac{1}{3 c_{1}}}+\Delta \sqrt{n}\right) \leq m^{1-c_{10}}
\end{aligned}
$$

for some constant $c_{10}>0$.

Therefore, there is a partition $V(G)=V_{1} \cup V_{2}$ such that $\left|V_{1}\right|,\left|V_{2}\right| \leq \frac{2}{3} n$ and $e_{G}\left(V_{1}, V_{2}\right) \leq m^{1-c_{10}}$. Since $G$ has $m$ edges in total, there is $i \in\{1,2\}$ such that $e_{G}\left(V_{i}\right) \geq \frac{\left|V_{i}\right|}{n}\left(m-m^{1-c_{10}}\right)$. Therefore, the subgraph of $G$ induced by $V_{i}$ has average degree at least a fraction $1-m^{-c_{10}} \geq 1-n^{-c_{10}}$ of the average degree of $G$. Letting $n_{1}=\left|V_{i}\right|$, we have $n_{1} \leq\left|V_{i}\right|$ and the subgraph of $G$ induced by $V_{i}$ also has maximum degree at most $\Delta$ and does not contain a $k$-grid with distinct vertices, completing the proof.

Repeatedly applying Lemma 2.6, we obtain the following lemma.

Lemma 2.7 Let $\Delta=(\log n)^{c \log k} k^{c \log \log k}$ as in Lemma 2.5. There is a constant $c^{\prime}$ such that $d_{k}\left(\Delta^{c^{\prime}}\right) \geq\left(1-\frac{1}{\Delta}\right) d_{k}(n, \Delta) \geq d_{k}(n, \Delta)-1$.

Proof. Let $n_{0}=n$. After one application of Lemma 2.6, we get $d_{k}\left(n_{1}, \Delta\right) \geq d_{k}(n, \Delta)\left(1-n^{-c_{10}}\right)$ for some $n_{1} \leq 2 n / 3$. After $j$ applications of Lemma 2.6, we get $d_{k}\left(n_{j}, \Delta\right) \geq d_{k}(n, \Delta) \prod_{i=1}^{j}\left(1-n_{i-1}^{-c_{10}}\right)$ for some $n_{j} \leq(2 / 3)^{j} n$. Let $i_{0}$ be the first value such that $n_{i_{0}} \leq \Delta^{c^{\prime}}$, where $c^{\prime}$ is a sufficiently large 
constant. We have

$$
\begin{aligned}
d_{k}\left(\Delta^{c^{\prime}}\right) & \geq d_{k}\left(\Delta^{c^{\prime}}, \Delta\right) \geq d_{k}\left(n_{i_{0}}, \Delta\right) \geq d_{k}(n, \Delta) \prod_{i=1}^{i_{0}}\left(1-n_{i-1}^{-c_{10}}\right) \geq d_{k}(n, \Delta)\left(1-\sum_{i=1}^{i_{0}} n_{i-1}^{-c_{10}}\right) \\
& \geq d_{k}(n, \Delta)\left(1-n_{i_{0}-1}^{-c_{10}} \sum_{i=0}^{\infty}(2 / 3)^{c_{10} i}\right) \geq d_{k}(n, \Delta)\left(1-n_{i_{0}-1}^{-c_{10}} \frac{1}{1-(2 / 3)^{c_{10}}}\right) \\
& \geq d_{k}(n, \Delta)\left(1-\left(\Delta^{c^{\prime}}\right)^{c_{10}} \frac{1}{1-(2 / 3)^{c_{10}}}\right) \geq d_{k}(n, \Delta)\left(1-\frac{1}{\Delta}\right) .
\end{aligned}
$$

By (2), we have $d_{k}(n) \leq(\log n)^{c_{3} \log 2 k}$. Since $c$ was chosen sufficiently large in Lemma 2.5, we have $d_{k}(n, \Delta) \leq d_{k}(n) \leq \Delta$. Summarizing,

$$
d_{k}\left(\Delta^{c^{\prime}}\right) \geq\left(1-\frac{1}{\Delta}\right) d_{k}(n, \Delta) \geq d_{k}(n, \Delta)-1
$$

The last inequality in Lemma 2.7 follows from (2) and the fact that the constant $c$ is chosen sufficiently large.

Combining Lemma 2.5, which gives us inequality (1), and Lemma 2.7 we therefore get that there is an absolute constant $C$ such that

$$
\begin{aligned}
& d_{k}\left((\log n)^{C \log k}\right) \geq d_{k}(n)-k^{C \log \log k} \quad \text { if } k \leq \log n \text {, and } \\
& d_{k}\left(k^{C \log \log k}\right) \geq d_{k}(n)-k^{C \log \log k} \quad \text { otherwise. }
\end{aligned}
$$

Iterating (3) until $n<\log k$, applying (4) if $k^{C \log \log k}<n<2^{k}$, and finally applying the trivial inequality $d_{k}(n) \leq n / 2$ if $n \leq k^{C \log \log k}$, we get that $d_{k}(n)=O\left(k^{C \log \log k} \log ^{*} n\right)$, and hence

$$
f_{k}(n)=O\left(k^{C \log \log k} n \log ^{*} n\right),
$$

completing the proof of Theorem 1.9.

\subsection{Topological graphs with no $(k, 1)$-grid with distinct vertices}

Let $G=(V, E)$ be a topological graph. For every edge $e \in E$ define $X(e)=\{f \in E \mid e$ crosses $f\}$. Given a set of edges $E^{\prime} \subset E$, the vertex cover number of $E^{\prime}$ is the minimum size of a set of vertices $V^{\prime} \subset V$ such that every edge in $E^{\prime}$ has at least one of its endpoints in $V^{\prime}$. Theorem 1.8 will follow from the next lemma, whose proof is due to Rom Pinchasi $[\mathrm{P}]$.

Lemma 2.8 Let $k$ be a fixed integer and let $G=(V, E)$ be a topological graph on $n$ vertices, such that for every $e \in E$ the vertex cover number of $X(e)$ is at most $k$. Then there is a constant $c_{k}$, such that $G$ has at most $c_{k} n$ edges.

Proof. We use a standard sampling argument. Let $m$ be the number of edges in $G$, and let $0<q<1$ be a constant. Let $G^{\prime}$ be the graph obtained from $G$ by taking every vertex of $G$ independently with probability $q$. Call an edge in $G^{\prime}$ good if it is not crossed by any other edge in $G^{\prime}$. Denote by $n^{*}$ and $m^{*}$ the expected number of vertices and good edges in $G^{\prime}$, respectively. Clearly, $n^{*}=q n$. The probability that an edge $e$ is good is at least $q^{2}(1-q)^{k}$, thus $m^{*} \geq q^{2}(1-q)^{k} m$. Since the good edges form a planar graph we have $q^{2}(1-q)^{k} m \leq m^{*} \leq 3 n^{*}=3 q n$. Thus, $m \leq \frac{3}{q(1-q)^{k}} n$. 
Now let $G$ be an $n$-vertex topological graph with no $(k, 1)$-grid with distinct vertices. We claim that for every $e \in E(G)$ the vertex cover number of $X(e)$ is at most $2 k$. Assume not. Then there is an edge $e \in E(G)$ such that the vertex cover number of $X(e)$ is at least $2 k+1$. Pick an edge $(u, v) \in X(e)$ and remove all the other edges in $X(e)$ that are covered by $v$ or $u$. This can be repeated $k$ times, for otherwise $X(e)$ can be covered by at most $2 k$ vertices. The edges we picked along with the edge $e$ form a $(k, 1)$-grid with distinct vertices. This proves Theorem 1.8.

\section{$3 \quad$ Natural grids in geometric and simple topological graphs}

In this section we consider natural grids in geometric and simple topological graphs and prove Theorems 1.4 and 1.5 .

\subsection{Proof of Theorem 1.4}

In this section we prove Theorem 1.4, which gives an upper bound on the number of edges of a geometric graph or a simple topological graph without a natural $k$-grid.

We use the following three results from three different papers. Pach et al. [PSS96] proved the following relationship between the crossing number and the bisection width of a graph.

Lemma 3.1 ([PSS96]) If $G$ is a graph with $n$ vertices of degrees $d_{1}, \ldots, d_{n}$, then

$$
b(G) \leq 7 \mathrm{cr}(G)^{1 / 2}+2 \sqrt{\sum_{i=1}^{n} d_{i}^{2}} .
$$

Let $m$ be the number of edges in $G$. Since $\sum_{i=1}^{n} d_{i}=2 m$ and $d_{i} \leq n$ for every $i$, we have

$$
b(G) \leq 7 \operatorname{cr}(G)^{1 / 2}+3 \sqrt{m n} .
$$

The following lemma is tight apart from the constant factor.

Lemma $3.2([\mathbf{F P 0 8 b}])$ For each $p$ there is a constant $c_{p}$ such that if $H$ is a graph with $n$ vertices, at least $c_{p}$ tn edges, and is an intersection graph of curves in the plane in which each pair of curves intersect in at most $p$ points, then $H$ contains the complete bipartite graph $K_{t, t}$ as a subgraph.

We will only need to use the case $p=1$. The last tool we use is an upper bound on the number of edges of a geometric graph with no $k$ pairwise disjoint edges.

Lemma 3.3 ([T00]) Any geometric graph with $n$ vertices and no $k$ pairwise disjoint edges has at most $2^{9}(k-1)^{2} n$ edges.

We now prove Theorem 1.4(i). As the proofs of (i) and (ii) are so similar, we only give the details for (i) and discuss how to modify the proof to obtain (ii).

Proof of Theorem 1.4(i): Let $g_{k}(n)$ be the maximum number of edges of a geometric graph with $n$ vertices and no natural $k$-grid. Let $G$ be a geometric graph on $n$ vertices and $m=g_{k}(n)$ edges with no natural $k$-grid. Let $c=\max \left(2^{20} c_{1}, 144\right)$, where $c_{1}$ is the constant with $p=1$ from Lemma 3.2. We prove by induction on $n$ that $g_{k}(n) \leq c k^{2} n \log ^{2} n$. Suppose for contradiction that $g_{k}(n)>c k^{2} n \log ^{2} n$. Let $\epsilon=10^{-3} \log ^{-2} n$. The proof splits into two cases, depending on whether or not the number of pairs of crossing edges of $G$ is less than $\epsilon m^{2}$. 
Case 1: The number of pairs of crossing edges is less than $\epsilon \mathrm{m}^{2}$. Then the crossing number of $G$ is less than $\epsilon m^{2}$. By (5), there is a partition $V(G)=V_{1} \cup V_{2}$ with $\left|V_{1}\right|,\left|V_{2}\right| \leq 2 n / 3$ and the number of edges with one vertex in $V_{1}$ and one vertex in $V_{2}$ is at most

$$
b(G) \leq 7 \mathrm{cr}(G)^{1 / 2}+3 \sqrt{m n} \leq 7 \epsilon^{1 / 2} m+3 \sqrt{m n}=\left(7 \epsilon^{1 / 2}+3 \sqrt{n / m}\right) m .
$$

Let $n_{1}=\left|V_{1}\right|$ and $n_{2}=\left|V_{2}\right|$, so $n=n_{1}+n_{2}$. Then we have

$$
\begin{aligned}
m & =g_{k}(n) \leq g_{k}\left(\left|V_{1}\right|\right)+g_{k}\left(\left|V_{2}\right|\right)+b(G) \leq g_{k}\left(n_{1}\right)+g_{k}\left(n_{2}\right)+\left(7 \epsilon^{1 / 2}+3 \sqrt{n / m}\right) m \\
& \leq c k^{2} n_{1} \log n_{1}+c k^{2} n_{2} \log n_{2}+\left(7 \epsilon^{1 / 2}+3 \sqrt{n / m}\right) m \leq c k^{2} n \log 2 n / 3+\left(7 \epsilon^{1 / 2}+3 \sqrt{n / m}\right) m \\
& \leq c k^{2} n \log n-c k^{2} n \log 3 / 2+\left(7 \epsilon^{1 / 2}+3 \sqrt{n / m}\right) m .
\end{aligned}
$$

This implies

$$
g_{k}(n)=m \leq c k^{2} n \frac{\log n-\log 3 / 2}{1-7 \epsilon^{1 / 2}-3 \sqrt{n / m}}<c k^{2} n \log n \frac{1-(\log 3 / 2)(\log n)^{-1}}{1-\left(\log ^{-1} n\right) / 4-3 c^{-1 / 2} k^{-1} \log ^{-1} n}<c k^{2} n \log n,
$$

where we use $3 c^{-1 / 2} k^{-1} \leq 1 / 4$. This completes the proof in this case.

Case 2: The number of pairs of crossing edges is at least $\mathrm{\epsilon m}^{2}$. Consider the intersection graph of the edges where two edges are adjacent if they cross. Since this intersection graph has $m$ vertices and at least $\epsilon \mathrm{m}^{2}$ edges and each pair of edges intersect at most once, Lemma 3.2 implies it contains a complete bipartite graph with parts of size

$$
t \geq \frac{\epsilon m}{c_{1}} \geq \frac{\log ^{-2} n m}{1000 c_{1}}>\frac{c}{1000 c_{1}} k^{2} n>2^{9} k^{2} n
$$

where $c_{1}$ is the constant with $p=1$ from Lemma 3.2. Therefore, $G$ contains edge subsets $E_{1}, E_{2}$ with $\left|E_{1}\right|=\left|E_{2}\right|=t$ and every edge in $E_{1}$ crosses every edge of $E_{2}$, i.e., $G$ contains a $t$-grid. Since $t>2^{9} k^{2} n$, Lemma 3.3 implies that $E_{i}$ contains $k$ disjoint edges for $i=1,2$. These two subsets of $k$ disjoint edges cross each other and hence form a natural $k$-grid, completing the proof.

To prove Theorem 1.4(ii), essentially the same proof work as above, except replacing the bound $O\left(k^{2} n\right)$ of Tóth [T00] on the number of edges of a geometric graph with no $k$ disjoint edges by the bound $O\left(n \log ^{4 k-8} n\right)$ of Pach and Tóth [PT05] on the number of edges of a simple topological graph with no $k$ disjoint edges.

\subsection{Natural (2,1)-grids: proof of Theorem $\mathbf{1 . 5}$}

Let $G=(V, E)$ be a simple topological graph on $n$ vertices without a natural $(2,1)$-grid. For every $e \in E$ assign $e$ the color red if $X(e)$ has vertex cover number at most 3, otherwise assign $e$ the color blue. It follows from Lemma 2.8 that $G$ has at most $29 n$ red edges (by picking $q=1 / 4$ ).

The proof of the next lemma is omitted due to space limitations. For $F \subseteq E$ denote by $V(F)$ the set of vertices induced by $F$.

Lemma 3.4 Let $e=(u, v)$ be a blue edge, and let $f_{1} \in X(e)$. Then if there is an edge $e^{\prime}=(u, w)$ such that $w \notin V(X(e))$ and $e^{\prime}$ crosses $f_{1}$, then $e^{\prime}$ crosses every edge $f \in X(e)$.

Next we remove all the red edges and process the blue edges in some arbitrary order. Let $B$ be the set of the currently unmarked and undeleted blue edges. Initially all the blue edges are in $B$. Let $e=(u, v)$ be the next edge in $B$. Delete all the edges that have one endpoint in $V(X(e) \cap B)$ and the other endpoint in $\{u, v\}$. Let $E_{u}$ be the edges $(u, x) \in B$ such that $x \notin V(X(e) \cap B)$ and 


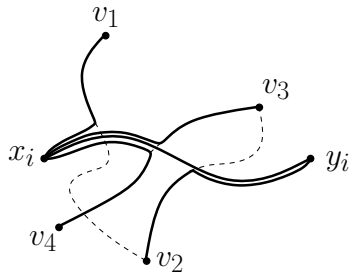

(a) Constructing $G^{\prime}$

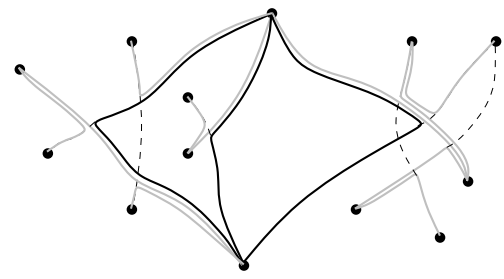

(b) $G^{\prime}$ might contain parallel edges

Figure 2: The graph $G^{\prime}$

there is an edge $e^{\prime} \in X(e) \cap B$ that crosses $(u, x)$. Similarly, let $E_{v}$ be the edges $(v, x) \in B$ such that $x \notin V(X(e) \cap B)$ and there is an edge $e^{\prime} \in X(e) \cap B$ that crosses $(v, x)$. Assume, w.l.o.g., that $\left|E_{u}\right| \geq\left|E_{v}\right|$ and remove the edges $E_{v}$. Recall that according to Lemma 3.4, if there is an edge $(u, x)$ such that $x \notin V(X(e))$, and $(u, x)$ crosses some edge in $X(e)$, then $(u, x)$ crosses every edge in $X(e)$.

A thrackle is a simple topological graph in which every pair of edges meet exactly once, either at a vertex or at a crossing point. It is known that a thrackle on $n$ vertices has at most $3(n-1) / 2$ edges [CN00] and it is a famous open problem (known as Conway's Thrackle Conjecture) to show that the tight bound is $n$. Set

$$
\operatorname{thrackle}(e)=B \cap\left(\{e\} \cup X(e) \cup\left\{(u, x) \mid \exists e^{\prime} \in X(e) \text { that } \operatorname{crosses}(u, v)\right\}\right)
$$

Mark all the blue edges in thrackle(e), and continue to create thrackles as long as there are unmarked blue edges. We omit the proofs of the next lemmas due to space limitations.

Lemma 3.5 thrackle(e) is a thrackle.

Lemma 3.6 If $e_{1} \in$ thrackle $(e)$ and $e_{2} \notin$ thrackle $(e)$ then $e_{1}$ and $e_{2}$ do not cross.

Since any newly created thrackle contains no edges of a previous thrackle, we obtain a partition of the edges that were not deleted into thrackles $t_{1}, t_{2}, \ldots, t_{j}$. Let $t_{i}=$ thrackle $\left(\left(u_{i}, v_{i}\right)\right)$ and denote by $V_{i}$ the vertex set of $t_{i}$. Recall that when $t_{i}$ was created at most $2\left|V_{i}\right|$ edges of the form $\left(x_{i}, y_{i}\right) \mid$ $x_{i} \in\left\{u_{i}, v_{i}\right\} \wedge y_{i} \in V\left(X\left(\left(u_{i}, v_{i}\right)\right)\right)$ and at most $\left|V_{i}\right|$ edges of the form $\left(x_{i}, y_{i}\right) \mid x_{i} \in\left\{u_{i}, v_{i}\right\} \wedge y_{i} \notin$ $V\left(X\left(\left(u_{i}, v_{i}\right)\right)\right)$ were deleted. The number of edges in $t_{i}$ is at most $3\left|V_{i}\right| / 2$, thus, it remains to show that $\sum_{i=1}^{j}\left|V_{i}\right|=O(n)$.

To this end we draw a new graph $G^{\prime}$ with the same vertex set $V$. For every thrackle $t_{i}=$ thrackle $\left(\left(x_{i}, y_{i}\right)\right)$ we draw a crossing-free tree $T_{i}$ with $\left|V_{i}\right|-1$ edges as follows. First, draw the edge from $x_{i}$ from $y_{i}$. Next, for every vertex $v \in V_{i} \backslash T_{i}$ pick one edge $e \in t_{i}$ that has $v$ as one of its endpoints. Follow $e$ from $v$ until it either hits a vertex (necessarily $x_{i}$ or $y_{i}$ ) or crosses an already drawn edge $e^{\prime}$. In the first case draw an edge identical to $e$. In the second case draw the segment of $e$ from $v$ almost until the crossing point, then continue the edge very close to $e^{\prime}$ (in one of the directions) until a vertex is reached. See Figure 2(a) for an example.

It follows from Lemma 3.6 and the construction of $G^{\prime}$ that $G^{\prime}$ is planar. Note that it is possible for $G^{\prime}$ to contain parallel edges (see Figure 2(b) for an example). However, it can be shown that they can be eliminated by removing at most half of the edges in $G^{\prime}$. We omit the details here. Therefore, $G^{\prime}$ has at most $6 n$ edges, and thus the number of edges in all the thrackles is at most $9 n$ and the total number of blue edges is at most $36 n$. We conclude that the original graph $G$ has at most $65 n$ edges. 


\section{Natural grids in convex geometric graphs}

In this section we first prove that an $n$-vertex convex geometric graph with no natural $k$-grid has $O(n)$ edges. Then, for convex geometric graphs avoiding natural $(2,1)-,(2,2)-$, or $(k, 1)$-grids we provide tighter bounds in terms of the constant hiding in the big- $O$ notation.

\subsection{Proof of Theorem $\mathbf{1 . 6}$}

We prove a stronger statement than Theorem 1.6, for which we need the following definition.

Definition 4.1 ([BKV03]) Given a convex geometric graph $G$, the cyclic chromatic number of $G$, is the minimum number of colors needed for a proper coloring of $G$ such that the vertices of every color class form a consecutive interval. $G$ is cyclic-bipartite if its cyclic chromatic number is at most 2.

Theorem 4.2 Let $k$ be a constant integer and let $D$ be a cyclic-bipartite convex geometric matching with $k$ edges. Then there is a constant $c_{k}$ such that any convex geometric graph on $n$ vertices that avoids $D$ has at most $c_{k} n$ edges.

Clearly a natural $k$-grid is a cyclic-bipartite convex geometric graph, hence, Theorem 4.2 implies Theorem 1.6. Moreover, Theorem 4.2 can be seen as a generalization of the Füredi-Hajnal Conjecture [FH92], that was settled by Marcus and Tardos [MT04].

Theorem 4.3 (Marcus-Tardos) Let $k$ be a constant. Then the number of 1-entries in any $n \times n$ 0 -1 matrix that avoids a given $k \times k$ permutation matrix ${ }^{1}$ is $O(n)$.

Indeed, a convex geometric graph is in fact a purely combinatorial object, since two edges cross if and only if their endpoints alternate in the cyclic order of the vertices. Thus, a 0-1 matrix can be seen as the adjacency matrix of a cyclic-bipartite convex geometric graph; and the Marcus-Tardos Theorem is equivalent to saying that every cyclic-bipartite convex geometric graph on $n$ vertices that avoids a cyclic-bipartite convex geometric matching of constant size has $O(n)$ edges. Therefore, Theorem 4.2 implies the Marcus-Tardos Theorem. We prove Theorem 4.2 by slightly modifying the proof of Marcus and Tardos. For details, see Appendix B.

\subsection{Tighter bounds for some special cases}

For specific values of $k$ or $l$ we are able to provide tighter bounds in terms of the constant $c_{k, l}$ for the number of edges in convex geometric graphs avoiding natural $(k, l)$-grids, than the ones guaranteed by Theorems 1.5 and 1.6. For the proofs, see Appendix C.

Theorem 4.4 An n-vertex convex geometric graph with no natural $(2,1)$-grid has less than $5 n$ edges.

Theorem 4.5 An n-vertex convex geometric graph with no natural $(2,2)$-grid has less than $8 n$ edges.

Theorem 4.6 A convex geometric graph with $n \geq 3 k$ vertices and no natural $(k, 1)$-grid has at most $6 k n-12 k^{2}$ edges.

Acknowledgments. We thank Rom Pinchasi for helpful discussions and for his permission to include his proof for Lemma 2.8 in this paper. The first author would also like to thank Balázs Keszegh for suggesting to consider $0-1$ matrices for the proof of Theorem 1.6.

\footnotetext{
${ }^{1} \mathrm{~A} k \times k 0-1$ matrix is a permutation matrix if it contains exactly one 1-entry in each row and column. A 0-1 matrix $A$ contains a $0-1$ matrix $B$ if one can delete some of its rows and columns and obtain a matrix of the same dimensions as $B$ that has a 1-entry in every place $B$ has a 1-entry. Otherwise, $A$ avoids $B$.
} 


\section{References}

[AKS98] P.K. Agarwal, M. van Kreveld, and S. Suri, Label placement by maximum independent set in rectangles, Comput. Geom. Theory Appl. 11 (1998), 209-218.

[BKV03] P. Brass, G. Károlyi, and P. Valtr, A Turán-type extremal theory for convex geometric graphs, Discrete and Computational Geometry - the Goodman-Pollack Festschrift (B. Aronov et al., eds.), Springer 2003, 275300 .

[CN00] G. Cairns and Y. Nikolayevsky, Bounds for generalized thrackles, Discrete and Computational Geometry 23 (2000), 191-206.

[CP92] V. Capoyleas and J. Pach, A Turán-type problem on chords of a convex polygon, J. Comb. Theory Ser. B. 56 (1992), 9-15.

[FP08] J. Fox and J. Pach, String graphs and incomparability graphs, manuscript, 2008.

[FP08a] J. Fox and J. Pach, Coloring $K_{k}$-free intersection graphs of geometric objects in the plane, 24nd ACM Symp. on Computational Geometry, College Park, MD, USA, June 2008, 346-354.

[FP08b] J. Fox and J. Pach, A separator theorem for string graphs and its applications, manuscript, 2008.

[FPT08] J. Fox, J. Pach, and Cs. D. Tóth, A bipartite strengthening of the Crossing Lemma, submitted, 2008.

[FH92] Z. Füredi and A. Hajnal, Davenport-Schinzel theory of matrices, Discrete Math. 103 (1992), 233-251.

[HM85] D.S. Hochbaum and W. Maass, Approximation schemes for covering and packing problems in image processing and VLSI, J. ACM 32 (1985), 130-136.

[KM04] P. Kolman and J. Matoušek, Crossing number, pair-crossing number, and expansion, J. Combin. Theory Ser. B. 92 (2004), 99-113.

[M97] E. Malesinska, Graph-theoretical models for frequency assignment problems, Ph.D. Thesis, Technische Universität Berlin, 1997.

[MT04] A. Marcus and G. Tardos, Excluded permutation matrices and the Stanley-Wilf conjecture, J. Comb. Theory Ser. A. 107 (2004), 153-160.

[PSS96] J. Pach, F. Shahrokhi, and M. Szegedy, Applications of the crossing number, Algorithmica 16 1996, 111-117.

[PPST05] J. Pach, R. Pinchasi, M. Sharir, and G. Tóth, Topological graphs with no large grids, Graphs and Combinatorics 21 (2005), 355-364.

[PT05] J. Pach and G. Tóth, Disjoint edges in topological graphs, in: Combinatorial Geometry and Graph Theory (J. Akiyama et al., eds.), Lecture Notes in Computer Science 3330, Springer-Verlag, Berlin, 2005, 133-140.

[PT94] J. Pach and J. Törőcsik, Some geometric applications of Dilworth's theorem, Discrete and Computational Geometry 12 (1994), 1-7.

[P] Rom Pinchasi, personal communication.

[T00] G. Tóth, Note on geometric graphs, J. Comb. Theory Ser. A. 89 (2000), 126-132.

[TT07] G. Tardos and G. Tóth, Crossing stars in topological graphs, SIAM Journal on Discrete Mathematics 21 (2007), 737-749. 


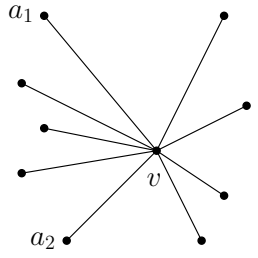

(a)

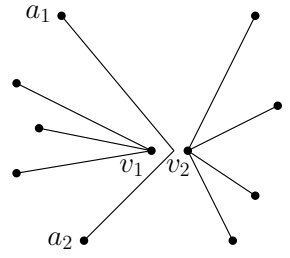

(b)
Figure 3: In (a) we have a vertex $v$ adjacent to two vertices $a_{1}, a_{2}$ which are not adjacent. In (b) we replace the edges $\left(a_{1}, v\right)$ and $\left(v, a_{2}\right)$ by one edge $\left(a_{1}, a_{2}\right)$ and split vertex $v$ into two vertices $v_{1}$ and $v_{2}$.

\section{A Proof of Lemma 2.5}

Proof. Let $G=(V, E)$ be a topological graph with $n$ vertices, $f_{k}(n)$ edges, and no $k$-grid with distinct vertices. Partition $V=A \cup B$, where $A$ consists of those vertices with degree more than $\Delta$. We construct a sequence of topological graphs $G_{i}$ and a sequence of vertex sets $B_{i}$ such that the vertex set of $G_{i}$ is $A \cup B_{i}$. Let $B_{0}=B$ and $G_{0}=G$. In graph $G_{i}$, if there is a vertex $v \in B_{i}$ adjacent to two vertices $a_{1}, a_{2} \in A$ which are not adjacent, then we replace the path of length two with edges $\left(a_{1}, v\right)$ and $\left(v, a_{2}\right)$ by an edge from $a_{1}$ to $a_{2}$, split the vertex $v \in B_{i}$ into two vertices $v_{1}, v_{2}$, set $B_{i+1}=\left\{v_{1}, v_{2}\right\} \cup B_{i} \backslash\{v\}$ and let $G_{i+1}$ be the resulting topological graph. See Figure 3 to see how $v$ is split into two vertices $v_{1}$ and $v_{2}$. We eventually stop at some step $j$ and we have a topological graph $G_{j}$ with vertex set $A \cup B_{j}$. For each vertex $v \in B_{j}$, the set $A_{v}$ of vertices in $A$ adjacent to $v$ form a clique in $G_{j}$, otherwise $v$ is adjacent to two vertices $a_{1}, a_{2} \in A$ that are not adjacent in $G_{j}$, which contradicts that we stopped at step $j$. Since $\left|B_{i+1}\right|=\left|B_{i}\right|+1$ for all $i$, we have $\left|B_{j}\right|=|B|+j$. The number of edges of $G_{j}$ is $j$ less than the number of edges of $G$ since $G_{i+1}$ is formed from $G_{i}$ by replacing a path of length two by an edge. Note that also the subgraph of $G_{j}$ induced by $A$ has $j$ more edges than the subgraph of $G$ induced by $A$.

We first provide an upper bound on the number of edges of $G_{j}$. Each edge in $G_{j}$ corresponds to either an edge or a path of length two in $G$. By construction, each pair of edges in $G_{j}$ that intersect come from two paths in $G$ (each path consisting of one or two edges) with distinct vertices. So if there are $h(k)$ pairwise intersecting edges in the subgraph of $G_{j}$ induced by $A$, then $G$ contains a $k$-grid with distinct vertices, a contradiction. By Lemmas 2.3 and 2.4, we have

$$
\begin{aligned}
e_{G}(A)+j & =e_{G_{j}}(A) \leq|A|(\log |A|)^{c_{3} \log h(k)} \\
& \leq|A|(\log n)^{c_{3} \log \left(c_{4} k \log k\right)} \leq|A|(\log n)^{c_{6} \log k}
\end{aligned}
$$

for some absolute constant $c_{6}$. In particular, $j \leq$ $|A|(\log n)^{c_{6} \log k}$.

As discussed above, for each vertex $v \in B_{j}$, the set $A_{v}$ of vertices form a clique in $G_{j}$. This clique can not have $h(k)$ pairwise intersecting edges, otherwise it contains a $k$-grid with distinct vertices. By Lemma 2.3 , we have

$$
\left(\begin{array}{c}
\left|A_{v}\right| \\
2
\end{array}\right) \leq\left|A_{v}\right|\left(\log \left|A_{v}\right|\right)^{c_{3} \log h(k)}
$$

so dividing both sides by $\left|A_{v}\right|$ we get

$$
\left|A_{v}\right| \leq 2\left(\log \left|A_{v}\right|\right)^{c_{3} \log h(k)}+1
$$

and finally

$$
\left|A_{v}\right| \leq h(k)^{c_{7} \log \log h(k)}
$$

for some absolute constant $c_{7}$. Also using Lemma 2.4, we have

$$
\left|A_{v}\right| \leq k^{c_{8} \log \log k}
$$

for some absolute constant $c_{8}$. So the number of edges $e_{G_{j}}\left(A, B_{j}\right)$ with one vertex in $A$ and the other vertex in $B_{j}$ is

$$
\sum_{v \in B_{j}}\left|A_{v}\right| \leq\left|B_{j}\right| k^{c_{8} \log \log k} .
$$

Since each vertex in $A$ has degree at least $\Delta$ in $G$, the number $e_{G}(A)+e_{G}(A, B)$ of edges in $G$ containing at least one vertex in $A$ is at least $|A| \Delta / 2$. So

$$
\begin{aligned}
|A| \Delta / 2 & \leq e_{G}(A)+e_{G}(A, B)=e_{G_{j}}(A)+e_{G_{j}}(A, B)+j \\
& \leq|A|(\log n)^{c_{6} \log k}+\left|B_{j}\right| k^{c_{8} \log \log k}+j \\
& =|A|(\log n)^{c_{6} \log k}+(|B|+j) k^{c_{8} \log \log k}+j \\
& \leq 2\left(|A|(\log n)^{c_{6} \log k}+n\right) k^{c_{8} \log \log k}
\end{aligned}
$$

If $n \leq|A|(\log n)^{c_{6} \log k}$, then we get

$$
\Delta \leq 8(\log n)^{c_{6} \log k} k^{c_{8} \log \log k},
$$

which contradicts $\Delta=(\log n)^{c \log k} k^{c \log \log k}$ with $c$ a sufficiently large constant. So $n>|A|(\log n)^{c_{6} \log k}$, and the number of edges in $G$ containing a vertex in $A$ is at most $4 k^{c 8} \log ^{\log k} n \leq k^{c \log \log k} n$. Note that every vertex in $B$ in $G$ has degree at most $\Delta$, so $e_{G}(B) \leq f_{k}(|B|, \Delta) \leq f_{k}(n, \Delta)$, where the last inequality follows by adding isolated vertices to $B$ to get a set of $n$ vertices. Therefore, the number $f_{k}(n)$ of edges of $G$ is at most $f_{k}(n, \Delta)+k^{c \log \log k} n$.

\section{B Proof of Theorem 4.2}

Proof. We slightly modify the Marcus-Tardos proof [MT04] of the Füredi-Hajnal conjecture.

Denote by $f(n, D)$ the maximum number of edges in a convex geometric graph that avoids $D$. Assume first that $n$ is divisible by $k^{2}$, and partition $V(G)$ into $n / k^{2}$ consecutive intervals, $I_{1}, I_{2}, \ldots, I_{n / k^{2}}$, each of size $k^{2}$. Denote by $E_{i, j}$ the set of edges between $I_{i}$ and $I_{j}$, and by $I_{i}\left(E_{i, j}\right)$ the set of vertices in $I_{i}$ that are endpoints of some edge in $E_{i, j}$. For $i \neq j$, we say that $I_{i}$ is dense with respect to $I_{j}$ if $\left|I_{i}\left(E_{i, j}\right)\right| \geq k$.

Lemma B.1 $I_{i}$ is dense with respect to less than $k\left(\begin{array}{c}k^{2} \\ k\end{array}\right)$ intervals. 


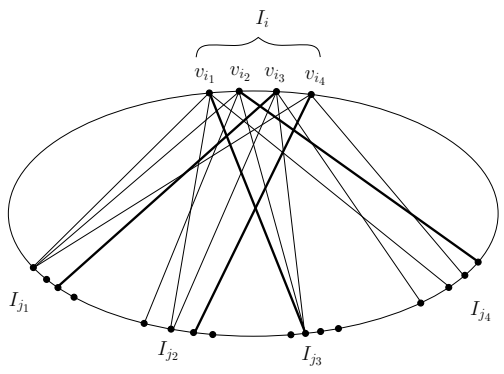

Figure 4: An illustration for the proof of Lemma B.1: realizing a natural $(2,2)$-grid

Proof. Assume that $I_{i}$ is dense with respect to at least $k\left(\begin{array}{c}k^{2} \\ k\end{array}\right)$ intervals. Then, by the pigeonhole principle, there is a set of $k$ of those intervals, $I_{j_{1}}, I_{j_{2}}, \ldots, I_{j_{k}}$, and a set of $k$ vertices $\left\{v_{i_{1}}, v_{i_{2}}, \ldots, v_{i_{k}}\right\} \subseteq I_{i}$, such that $\left\{v_{i_{1}}, v_{i_{2}}, \ldots, v_{i_{k}}\right\} \subseteq I_{i}\left(E_{i, j_{1}}\right) \cap I_{i}\left(E_{i, j_{2}}\right) \cap \cdots \cap I_{i}\left(E_{i, j_{k}}\right)$. The set of edges $E_{i, j_{1}} \cup E_{i, j_{2}} \cup \cdots \cup E_{i, j_{k}}$, reduced to the vertices $v_{i_{1}}, v_{i_{2}}, \ldots, v_{i_{k}}$, can be used to realize any cyclic-bipartite convex geometric matching with $k$ edges (see Figure 4 for an example).

Construct a new convex geometric graph $G^{\prime}$, by contracting every interval $I_{i}$ of $G$ into a single vertex $u_{i}$, and drawing an edge between $u_{i}$ and $u_{j}$ if and only if there are vertices $v_{i} \in I_{i}$ and $v_{j} \in I_{j}$ such that $\left(v_{i}, v_{j}\right) \in E(G)$. Observe that $G^{\prime}$ avoids $D$, for otherwise $G$ must contain $D$.

We can now upper-bound $f(n, D)$ by the expression:

$$
\begin{gathered}
\sum_{i}\left|E_{i, i}\right|+\sum_{\substack{i \neq j \\
I_{i} \text { dense w.r.t. } I_{j}}}\left|E_{i, j}\right|+\sum_{\substack{i \neq j \\
I_{i} \text { not dense w.r.t. } I_{j} \\
I_{j} \text { not dense w.r.t. } I_{i}}}\left|E_{i, j}\right| \\
\leq\left(\begin{array}{c}
k^{2} \\
2
\end{array}\right) \frac{n}{k^{2}}+k^{4} \cdot k\left(\begin{array}{c}
k^{2} \\
k
\end{array}\right) \frac{n}{k^{2}}+(k-1)^{2} \cdot f\left(\frac{n}{k^{2}}, D\right) \\
\leq 2 k^{3}\left(\begin{array}{c}
k^{2} \\
k
\end{array}\right) n+(k-1)^{2} \cdot f\left(\frac{n}{k^{2}}, D\right)
\end{gathered}
$$

Using this recursive bound, Theorem 4.2 easily follows by induction on $n$ for $k \geq 2$ with $c_{k}=2 k^{4}\left(\begin{array}{c}k^{2} \\ k\end{array}\right)$ (see [MT04, Theorem 8]).

\section{Proofs of Theorems 4.4, 4.5, and 4.6}

We mention first some basic notions and facts before moving to the proofs. Let $G$ be a convex geometric graph. We denote by $d_{G}(v)$ the degree of a vertex $v$ in $G$, and by $\delta(G)$ the minimum degree in $G$. For $u, v \in V(G)$, we say that $v$ and $u$ are consecutive vertices if they appear next to each other on the convex hull of the vertices of $G$. For $u, v \in V(G)$ we denote by $R(u, v) \subset V(G)$ the set of vertices from $u$ to $v$ in clockwise order, not including $u$ and $v$.
A convex geometric graph $G^{\prime}$ is a geometric minor of $G$ if $G^{\prime}$ can be obtained from $G$ by performing a finite number of the following two operations:

1. Vertex deletion.

2. Consecutive vertex contraction, i.e., only consecutive vertices can contract. Recall that the contraction of two vertices $x$ and $y$, replaces $x$ and $y$ in $G$ with a vertex $v$, such that $v$ is adjacent to all the neighbors of $x$ and $y$.

Notice that if two edges $e_{1}$ and $e_{2}$ cross in $G^{\prime}$, then they cross in $G$. Likewise, if $e_{1}$ and $e_{2}$ are disjoint in $G^{\prime}$, then they are disjoint in $G$. Assume that $G$ is a convex geometric graph with $n$ vertices and at least $c n$ edges. Let $G^{\prime}$ be a minimal geometric-minor of $G$ such that $\left|E\left(G^{\prime}\right)\right| /\left|V\left(G^{\prime}\right)\right| \geq$ c. Then we can conclude that:

1. $\delta\left(G^{\prime}\right) \geq c$ (otherwise vertex deletion can be applied); and

2. every consecutive pair of vertices $v$ and $u$ must have at least $c-1$ common neighbors (otherwise consecutive vertex contraction can by applied).

Proof of Theorem 4.4: Suppose that $|E(G)| \geq 5 n$. Let $G^{\prime}$ be a minimal geometric-minor of $G$ such that $\left|E\left(G^{\prime}\right)\right| /\left|V\left(G^{\prime}\right)\right| \geq 5$. Note that $\left|V\left(G^{\prime}\right)\right| \geq 11$. For a vertex $u \in V\left(G^{\prime}\right)$ denote by $u_{1}, u_{2}, \ldots$ the neighbors of $u$ in clockwise order. Note that $u_{1}$ immediately follows $u$ in clockwise order, since a straight-line segment connecting two consecutive vertices in $G$ cannot be crossed by any edge of $G$ and hence we can assume w.l.o.g. that it is an edge of $G$. Let $v \in V\left(G^{\prime}\right)$ be the vertex such that:

$$
\left|R\left(v_{3}, v\right)\right|=\min _{u \in V\left(G^{\prime}\right)}\left|R\left(u_{3}, u\right)\right|
$$

Since $\delta\left(G^{\prime}\right) \geq 5, u_{3}$ exists for every $u$. Since $v_{1}$ and $v$ are consecutive vertices they share at least 4 common neighbors. Hence $v_{1}$ and $v$ are both adjacent to a vertex $a \in V\left(G^{\prime}\right)$, such that $a \notin\left\{v_{2}, v_{k-1}, v_{k}\right\}$, where $k=d_{G^{\prime}}(v)$. By minimality of $\left|R\left(v_{3}, v\right)\right|, v_{k}$ has at least three neighbors in $R\left(v_{k}, v_{3}\right)$. Thus $v_{k}$ has a neighbor $b \in R\left(v_{k}, v_{3}\right)$ other than $v$ and $v_{1}$. Hence we have a natural $(2,1)$-grid with edges $\left(v, v_{k-1}\right),\left(v_{1}, a\right)$, and $\left(v_{k}, b\right)$ in $G^{\prime}$, and hence in $G$.

Proof of Theorem 4.5: Assume that $|E(G)| \geq$ $8 n$. Let $G^{\prime}$ be a minimal geometric-minor of $G$ with $\left|E\left(G^{\prime}\right)\right| /\left|V\left(G^{\prime}\right)\right| \geq 8$. Note that $\left|V\left(G^{\prime}\right)\right| \geq 17, \delta\left(G^{\prime}\right) \geq 8$, and every pair of consecutive vertices in $G^{\prime}$ share at least 7 common neighbors. Let $\left(x, x^{\prime}\right)$ and $\left(y, y^{\prime}\right)$ be a pair of disjoint edges such that:

1. $x$ and $y$ are consecutive vertices with $x$ following $y$ in clockwise order;

2. $\left|R\left(x, x^{\prime}\right)\right|,\left|R\left(y^{\prime}, y\right)\right| \geq 2$; and

3. $\left|R\left(y^{\prime}, y\right)\right|$ is maximized subject to (1) and (2) above.

This is possible since consecutive vertices share at least 7 common neighbors. Now let $u, v$ be the next two vertices after $x$ in clockwise order. Since $u$ and $v$ are consecutive, 


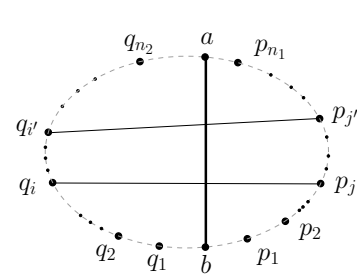

(a) $q_{i} p_{j} \prec q_{i^{\prime}} p_{j^{\prime}}$

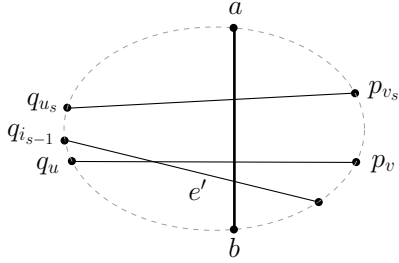

(b) The second case in the proof of Proposition C.1

Figure 5: Illustrations for the proof of Theorem 4.6
1. $u_{t}=i_{t}$.

2. $u_{x} \geq i_{x}$ for $x=1, \ldots, t-1$.

3. $\operatorname{rank}\left(q_{u_{x}} p_{v_{x}}\right)=r_{x}$, for $x=1, \ldots, t$.

Proof. By reverse induction on $x$. In $G$ we can pick the edge $q_{i_{t}} p_{v_{t}}$ that has rank $r_{t}$. We know one exists since $q_{i_{t}} p_{r_{t}}^{*}$ exists in $G_{1}$. Assume that we have already found the edges $q_{u_{x}} p_{v_{x}}$ for $x=t, t-1, \ldots, s>1$ that satisfy the above. Let $q_{u} p_{v}$ be an edge of rank $r_{s-1}$ such that $q_{u} p_{v} \prec q_{u_{s}} p_{v_{s}}$. If $u \geq i_{s-1}$, then we can pick $q_{u} p_{v}$ as the next edge. Otherwise, let $e^{\prime}$ be an edge of rank $r_{s-1}$ with $q_{i_{s-1}}$ as an endpoint. Since $e^{\prime}$ and $q_{u} p_{v}$ have the same rank, they must cross, which implies that $e^{\prime} \prec q_{u_{s}} p_{v_{s}}$ and so we can pick $e^{\prime}$ as the next edge. See Figure 5(b).

Proposition C.2 $G_{1}$ does not contain a natural $(k, 1)$ grid.

Proof. Assume that $G_{1}$ contains a natural $(k, 1)$-grid. Then by considering the possible edges involved in such a grid and using Proposition C.1 above, one concludes that there is a natural $(k, 1)$-grid in $G$, which is a contradiction.

We also define a convex geometric graph $G_{2}$ with $n_{1}+$ $k+1$ vertices $\left\{a, p_{n_{1}}, \ldots, p_{1}, b, q_{1}^{*}, \ldots, q_{k-1}^{*}\right\}$ (in clockwise order). Let $G_{2}$ be the same as $G$ when restricted to the vertices $\left\{a, p_{n_{1}}, \ldots, p_{1}, b\right\}$. Then let $\left(q_{r}^{*}, p_{j}\right)$ be in $E\left(G_{2}\right)$ if and only if there is an edge $\left(q_{i}, p_{j}\right) \in E(G)$ whose rank is $r$. By the same arguments $G_{2}$ does not contain a natural $(k, 1)$-grid. Let $E_{r}$ denote the edges in $G$ with rank $r, 1 \leq$ $r \leq k-1$.

Proposition C.3 $\left|E_{r}\right| \leq d_{G_{1}}\left(p_{r}^{*}\right)+d_{G_{2}}\left(q_{r}^{*}\right)-1$.

Proof. The edges in $E_{r}$ cannot form a cycle. Indeed, consider a path $q_{i_{1}} p_{j_{1}}, q_{i_{2}} p_{j_{1}}, q_{i_{2}} p_{j_{2}}, \ldots$ and assume w.l.o.g. that $i_{1}<i_{2}$. Then $j_{2}<j_{1}$ for otherwise $q_{i_{1}} p_{j_{1}}$ and $q_{i_{2}} p_{j_{2}}$ are disjoint. Similarly, we have $i_{l}>i_{l-1}$ and $j_{l}<j_{l-1}$, for any $l>1$, therefore the path can not form a cycle. Since there are $d_{G_{1}}\left(p_{r}^{*}\right)+d_{G_{2}}\left(q_{r}^{*}\right)$ vertices that are endpoints of edges in $E_{r}$, the claim follows.

Denote by $E_{1}^{\prime}$ the edges in $G_{1}$ that do not cross $a b$ and by $E_{2}^{\prime}$ the edges in $G_{2}$ that do not cross $a b$ (note that $\left.a b \in E_{i}^{\prime}, i=1,2\right)$. Recall that $a b$ has at least $2 k$ vertices on each of its sides, therefore, $\left|V\left(G_{1}\right)\right|,\left|V\left(G_{2}\right)\right| \geq 3 k$. Then:

$$
\begin{aligned}
|E(G)|= & \left|E_{1}^{\prime}\right|+\left|E_{2}^{\prime}\right|-1+\sum_{r=1}^{k-1}\left|E_{r}\right| \\
& =\quad\left|E_{1}^{\prime}\right|+\left|E_{2}^{\prime}\right|-1+\sum_{r=1}^{k-1}\left(d_{G_{1}}\left(p_{r}^{*}\right)+d_{G_{2}}\left(q_{r}^{*}\right)-1\right) \\
= & \left|E\left(G_{1}\right)\right|+\left|E\left(G_{2}\right)\right|-k \\
\text { ind hyp } & \left(6 k\left(n_{1}+k+1\right)-12 k^{2}\right) \\
\leq & +\left(6 k\left(n_{2}+k+1\right)-12 k^{2}\right)-k \\
= & 6 k n-12 k^{2}-k \leq 6 k n-12 k^{2}
\end{aligned}
$$

This completes the proof of Theorem 4.6. joint edges in $G_{1}$ that cross ab. Then there are $t$ pairwise disjoint edges $q_{u_{1}} p_{v_{1}}, \ldots, q_{u_{t}} p_{v_{t}}$ such that 